\title{
Caracterização da madeira laminada colada de Eucalyptus sp. produzida com adesivos resorcinol-fenol-formaldeído e poliuretano
}

\author{
Pedro Gutemberg de Alcântara Segundinho*, Alaor Coelho Silva, Fabrício Gomes Gonçalves, \\ Adair José Regazzi
}

Departamento de Ciências Florestais e da Madeira, Universidade Federal do Espírito Santo (UFES), Jerônimo Monteiro, ES, Brasil.

\begin{abstract}
RESUMO A fabricação e a utilização de madeira laminada colada (MLC) são consolidadas há muito tempo em países da Europa e da América do Norte utilizando madeira de pinus, para atender o mercado da construção civil no fornecimento de elementos estruturais. O objetivo deste trabalho foi avaliar as propriedades físicas e mecânicas da madeira de Eucalyptus sp., visando à produção de elementos de MLC colados com adesivos à base de resorcinol-fenol-formaldeído (RFF) e poliuretano (PUR). A partir de corpos de prova obtidos de elementos de MLC, investigou-se a resistência mecânica aos esforços de compressão paralela às fibras, compressão normal às fibras, cisalhamento nas linhas de cola, além da comparação dos resultados com os corpos de prova obtidos de madeira maciça, avaliação da eficiência das linhas de cola por meio de ensaio de delaminação e penetração dos adesivos na madeira. As madeiras coladas com RFF apresentaram maior espessura de linha de cola, menor delaminação da linha de cola, além de uma resistência maior no cisalhamento, em comparação a madeira colada com PUR. Logo, foi possível concluir que a madeira de Eucalyptus sp. associada ao adesivo RFF possui grande potencial na fabricação de MLC, principalmente para ambiente externo sujeito a intempéries.
\end{abstract}

Palavras-chave: Elementos estruturais; propriedades físicas; propriedades mecânicas; espessura de linha de cola; delaminação.

\section{Characterization of glued-laminated timber from Eucalyptus sp. produced with resorcinol-phenol-formaldehyde and polyurethane adhesives}

\begin{abstract}
The manufacture and use of glued-laminated timber (Glulam) have been consolidated for a long time in European and North American countries, where pine wood has been used to supply the construction market with structural elements. The aim of this study was to evaluate the physical and mechanical properties of Eucalyptus sp. wood, for the production of Glulam elements glued with adhesives based on resorcinol-phenol-formaldehyde (RPF) and polyurethane (PUR). From specimens obtained from Glulam elements, the mechanical resistance to parallel compression efforts on fibers, normal fiber compression, shear on glue lines were investigated; and the comparison of results with specimens from solid wood, the assessment of glue lines efficiency through the delamination test, and penetration of adhesives in wood. Glued timber with RPF presented thicker glue line, less delamination in the glue line, and a larger shear resistance, compared to the beams glued with PUR. Thus, it was possible to conclude that Eucalyptus sp. wood associated with the RPF adhesive has great potential in the manufacturing of Glulam, especially for outdoors subjected to undesirable weather.
\end{abstract}

Keywords: Structural elements; physical properties; mechanical properties; thickness of glue line; delamination.

\section{Introdução}

A madeira laminada colada (MLC) é um produto estrutural obtido a partir da utilização de duas técnicas: a técnica de lamelar e a técnica de colar. A colagem é realizada em peças de madeira denominadas lamelas, de modo a formar o elemento estrutural. A altura desejada do elemento 
estrutural de MLC é obtida pela colagem face a face destas lamelas; na forma tradicional, para facilitar a produção dos elementos estruturais, as fibras da madeira são dispostas paralelamente ao eixo longitudinal.

A MLC surgiu no século passado, em Wiemar, Alemanha, a partir dos testes iniciais de Otto Karl Frederich Hetzer, que desenvolveu essa técnica de construção e obteve a primeira patente. Em 1906, Hetzer patenteou na Alemanha as construções de MLC na forma curva e, desde então, tal técnica (inicialmente chamada de Estruturas Hetzer) vem sendo utilizada por diversos países do mundo. As aplicações de MLC destinavam-se primeiramente a sistemas estruturais interiores; no entanto, o desenvolvimento de adesivos à prova de água permitiu que a MLC passasse a ser utilizada com sucesso em estruturas expostas a intempéries, viabilizando a construção de coberturas, pontes e elementos estruturais, como vigas, colunas, dormentes e cruzetas (ICIMOTO et al., 2016).

As associações American Institute of Timber Construction (AITC) e American Plywood Association (APA) representam a maioria dos produtores de MLC da América do Norte e são responsáveis por assegurarem aos consumidores a qualidade da MLC por meio da divulgação das instruções normativas contidas na Standard for Adhesives for Use in Structural Glued Laminated Timber (ANSI 405, 2013), Standard Specification for Structural Glued Laminated Timber of Softwood Species (ANSI 117, 2015) e Standard for Wood Products - Structural Glued Laminated Timber (ANSI A190.1, 2017).

A redução da disponibilidade de espécies de madeiras nativas tornou necessário o desenvolvimento de técnicas alternativas viáveis para a utilização racional da madeira proveniente de florestas plantadas. Diante desse cenário, a MLC vem sendo mais usada em diversos países por apresentar versatilidade no que diz respeito às formas, dimensões e resistência mecânica dos elementos estruturais, aliados ao aumento de durabilidade, proporcionada pelos tratamentos preservativos.

Nas últimas décadas, os elementos estruturais derivados de madeira, tal como a MLC, têm propiciado novos campos de aplicação para esse material, uma vez que apresentam padrões compatíveis com as modernas exigências das construções. A redução progressiva dos estoques de madeira serrada e a necessidade de racionalização do seu uso incentivam o desenvolvimento de novas soluções estruturais que, preservando no produto a beleza do material original, permitem aumentar o seu desempenho e, consequentemente, reduzir o seu consumo (APA, 2008).

A maioria dos elementos estruturais em MLC no Brasil é produzida principalmente com a madeira de pinus (TEREZO; SZÜCS, 2010); além da produção com a madeira de pinus, também são confeccionadas vigas com a madeira de eucalipto, as quais são espécies exóticas que não pertencem à flora nativa brasileira (TEREZO; SZÜCS, 2010). Por ser proveniente de florestas plantadas e devido a suas propriedades físicas e mecânicas, a madeira de Eucalyptus sp. surge com potencial para ser utilizada na fabricação de MLC.

O desenvolvimento da MLC no Brasil é de grande importância para o emprego da madeira na construção civil, pois apresenta inúmeras vantagens, tais como uma construção mais rápida, eficiente e versátil. No Brasil, o uso da MLC iniciou-se na década de 40 com a tecnologia trazida por alemães; possivelmente a primeira indústria a utilizá-la foi a Esmara Estruturas de Madeira Ltda., fundada em 1954, em Porto Alegre, Rio Grande do Sul.

O presente estudo teve como objetivo avaliar as propriedades físicas e mecânicas da MLC produzida com madeira de Eucalyptus sp. e colada com adesivos à base de resorcinol-fenol-formaldeído e poliuretano. O estudo possibilitou uma análise da qualidade desses adesivos tanto na condição seca quanto saturada. 


\section{Material e Métodos}

\section{A madeira}

O presente estudo foi conduzido no Departamento de Ciências Florestais e da Madeira da Universidade Federal do Espírito Santo, em Jerônimo Monteiro, Espírito Santo. Para a fabricação das lamelas que compõem os elementos de MLC foram utilizadas 24 tábuas de madeira de Eucalyptus sp., sendo 12 tábuas para o tratamento L1, lote de madeira utilizada para colagem com poliuretano e oriundo do município de Jerônimo Monteiro, e 12 tábuas para o tratamento L2, lote de madeira utilizada para colagem com resorcinol-fenol-formaldeido e oriundo do município de Alegre. Tais municípios estão localizados na região sul do Estado do Espirito Santo.

A madeira de Eucalyptus sp. dos lotes L1 e L2 teve origem de reflorestamento (madeira de ciclo curto na faixa de 7 a 10 anos) e encontrava-se seca. No entanto, foram devidamente estocadas na marcenaria do departamento durante um período de três meses, os quais alcançaram os seguintes teores de umidade de equilíbrio com o ambiente: L1 igual a 11,80\% e L2 igual a $14,33 \%$.

\section{Confecção dos elementos de MLC}

Para a execução da pesquisa foram fabricados 20 elementos de MLC - 10 colados com adesivo poliuretano originando o tratamento MLC-PUR-L1 e 10 colados com adesivo resorcinol-fenol-formaldeído originando o tratamento MLC-RFF-L2 -, que juntos consumiram 80 lamelas nas dimensões $530 \mathrm{~mm}$ x $90 \mathrm{~mm}$ x 22,5 mm (comprimento $\mathrm{x}$ largura $\mathrm{x}$ espessura). As lamelas passaram por uma inspeção visual a fim de se identificar defeitos anatômicos ou imperfeições provenientes da secagem, como esmoados, nós e rachaduras de topo. Em cada prensagem foram fabricados quatro elementos de MLC, sendo utilizadas
16 lamelas de madeira. As lamelas foram usinadas com uma desengrossadeira no dia da colagem para evitar a obstrução dos poros da madeira e seu empenamento, o que contribui para a redução da resistência da linha de cola.

Os adesivos foram preparados a uma temperatura de aproximadamente $25^{\circ} \mathrm{C}$ e posteriormente espalhados sobre a superfície da lamela com o auxílio de uma espátula. Os dois tratamentos foram colados com gramatura igual a 300 g.m $\mathrm{m}^{-2} \mathrm{e}$ pressão de colagem igual a 1,0 $\mathrm{MPa}$. Tais parâmetros de colagem atendem as recomendações estabelecidas pelos fabricantes, mesmo sendo adesivos diferentes.

As lamelas foram unidas com o auxílio de uma prensa com capacidade de 15 toneladas, mantidas por $72 \mathrm{~h}$ (Figura 1A) em um ambiente com temperatura igual a $20^{\circ} \mathrm{C}$ e umidade relativa do ar igual a $65 \%$, conforme recomendação da NBR 7190 (ABNT, 1997). Após o decorrer desse tempo, os elementos foram retirados e mantidos em repouso durante 10 dias para finalização da secagem e aderência da cola (Figura 1B).

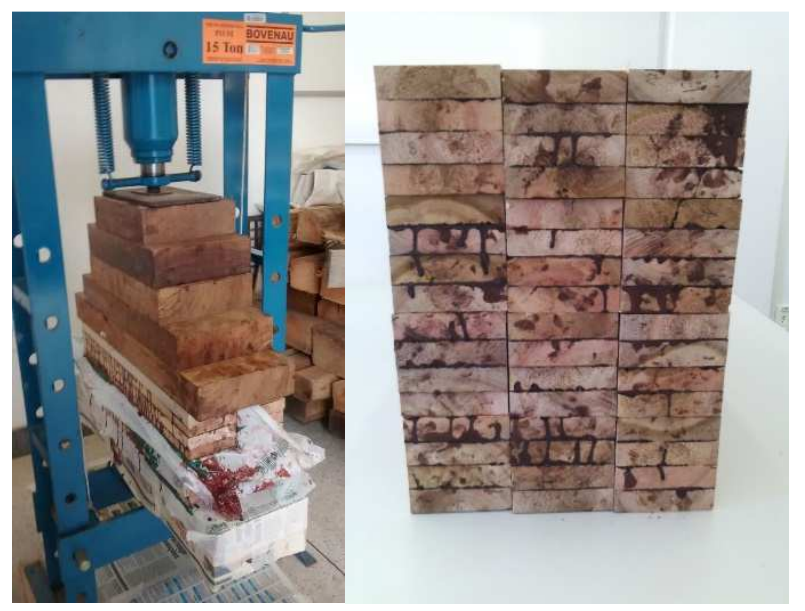

Figura 1. A) Prensagem dos elementos de MLC; B) Elementos de MLC finalizados.

Figure 1. A) Pressing of the Glulam elements; B) Glulam elements finalized. 


\section{Caracterização das propriedades físicas e} mecânicas da madeira maciça

A caracterização das propriedades físicas da madeira maciça dos dois lotes, compostos por 12 tábuas cada lote, foi conduzida de acordo com os métodos de ensaio definidos no Anexo B da NBR 7190 (ABNT, 1997), para a determinação do teor de umidade $(\mathrm{U})$, da densidade aparente $\left(\rho_{\text {app }}\right)$, da densidade básica $\left(\rho_{\text {bas }}\right)$, da retração volumétrica $(R V)$ e do inchamento volumétrico (IV).

Para a caracterização das propriedades mecânicas da madeira maciça dos dois lotes, determinação da resistência à compressão paralela às fibras $\left(\mathrm{f}_{\mathrm{c} 0}\right)$ e determinação da resistência ao cisalhamento paralelo às fibras $\left(f_{\mathrm{v} 0}\right)$, foram aplicados os métodos de ensaio da norma American Society for Testing and Materials - ASTM D143 - Standard Test Methods for Small Clear Specimens of Timber (ASTM, 2000). O equipamento utilizado na realização dos ensaios mecânicos foi uma máquina universal de ensaios (EMIC - Linha $D L$ 10.000) com capacidade de $100 \mathrm{kN}$ e sistema automatizado de obtenção de dados.

\section{Caracterização das propriedades mecânicas da}

\section{$M L C$}

A caracterização das propriedades mecânicas dos 20 elementos de MLC foi realizada a partir de corpos-de-prova de compressão paralela às fibras, cisalhamento na linha de cola, compressão normal às fibras, delaminação e corpos-deprova de compressão normal às fibras denominados estruturais porque representam uma seção transversal do bloco, conforme Figura 2. As amostras foram divididas em duas condições de ensaios. Na primeira condição, as amostras foram mantidas com teor de umidade em equilíbrio com o ambiente e são consideradas secas; na segunda condição, as amostras foram imersas em água por um período mínimo de duas semanas com o objetivo de elevar o teor de umidade da madeira para acima do ponto saturação das fibras (U>PSF) e são consideradas saturadas. Os corpos-de-prova foram extraídos ao longo do comprimento dos elementos de MLC de forma planejada (Figura 2). O equipamento utilizado na realização dos ensaios mecânicos foi uma máquina universal de ensaios (EMIC - Linha DL 10.000) com capacidade de 100 $\mathrm{kN}$ e sistema automatizado de obtenção de dados.

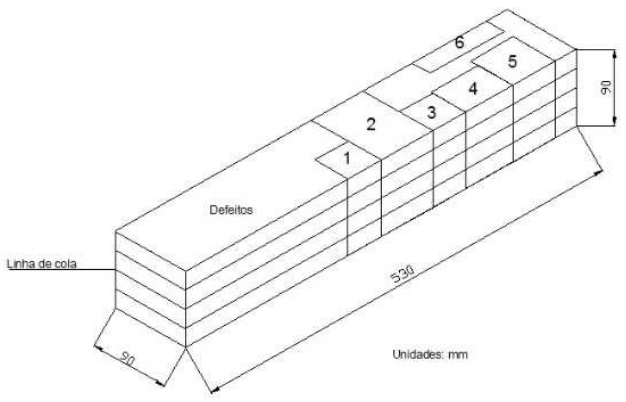

Figura 2. Esquema de retirada dos corpos-de-prova do bloco de MLC, sendo a área 1 destinada ao corpo de prova estrutural, 2 e 4 destinadas ao corpo de prova de compressão normal às fibras, 3 à delaminação, 5 ao cisalhamento na linha de cola, e 6 à compressão paralela às fibras.

Figure 2. Scheme to cut specimens from the Glulam block; area 1 was destined for structural specimens, areas 2 and 4 were destined to normal fiber compression tests, area 3 to delamination, area 5 to glue line shear, and area 6 to compression parallel to the fibers.

\section{Ensaio de cisalhamento da linha de cola da MLC}

Para o ensaio e determinação da resistência ao cisalhamento na linha de cola $\left(\mathrm{f}_{\mathrm{v} 0}\right)$, o procedimento de teste foi realizado de acordo com a norma ASTM D 905 - Test Method for Strength Properties of Adhesive Bonds in Shear by Compression Loading (ASTM, 2009).

A avaliação da falha na madeira foi analisada após a execução do ensaio de resistência ao cisalhamento na linha de cola $\left(f_{v 0}\right)$. A inspeção da porcentagem de cisalhamento na madeira ocorreu na área da linha de cola e foi realizada com o auxílio de uma malha composta por cem partes iguais (Figura 3), transcrita em uma transparência e sobreposta ao 
corpo de prova. A porcentagem de falha na madeira foi obtida a partir da contagem dos espaços na malha onde ocorreu cisalhamento na madeira, conforme a norma ASTM D 905 Test Method for Strength Properties of Adhesive Bonds in Shear by Compression Loading (ASTM, 2009).

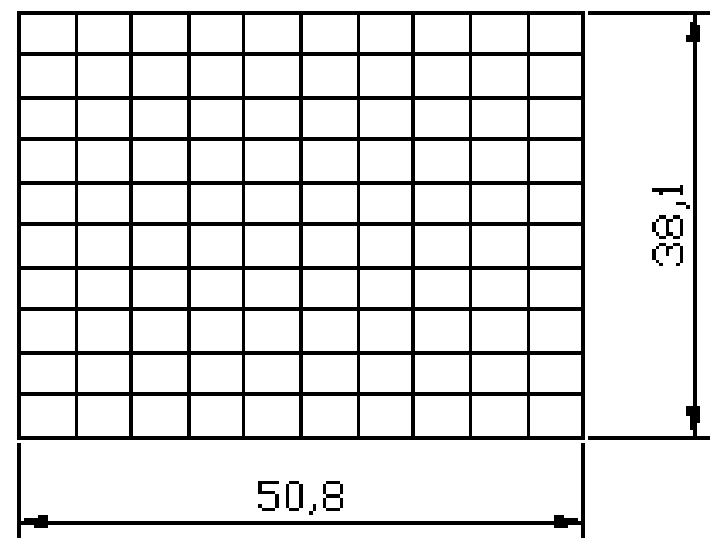

Figura 3. Gabarito para análise de falha na madeira (unidade em $\mathrm{mm})$.

Figure 3. Feedback for the analysis of failure on wood (units in $\mathrm{mm}$ ).

\section{Ensaio de compressão normal às fibras na MLC}

As dimensões do corpo-de-prova de compressão normal às fibras, obtidos a partir dos elementos de MLC, para realização dos ensaios secos e saturados, podem ser verificadas na Figura 4. O procedimento de ensaio foi realizado conforme a norma ASTM D143 - Standard Test Methods for Small Clear Specimens of Timber (ASTM, 2000). A resistência $\left(\mathrm{f}_{\mathrm{c} 90}\right)$ e o módulo de elasticidade $\left(\mathrm{E}_{\mathrm{c} 90}\right)$ à compressão normal às fibras foram obtidos de acordo com as normas ISO 13910 (2005) e NBR 7190 (ABNT, 1997), respectivamente. As pesquisas atuais em andamento no Brasil levam a crer que tais normas são mais adequadas para obtenção dessas propriedades físicas.

\section{Ensaio de delaminação da MLC}

A MLC também foi avaliada por meio do ensaio de delaminação utilizando o corpo-de-prova apresentado na
Figura 5, segundo a recomendação AITC T110 (2007). Tal ensaio possibilita avaliar o comportamento da combinação espécie-adesivo para uso exterior mediante autoclavagem.

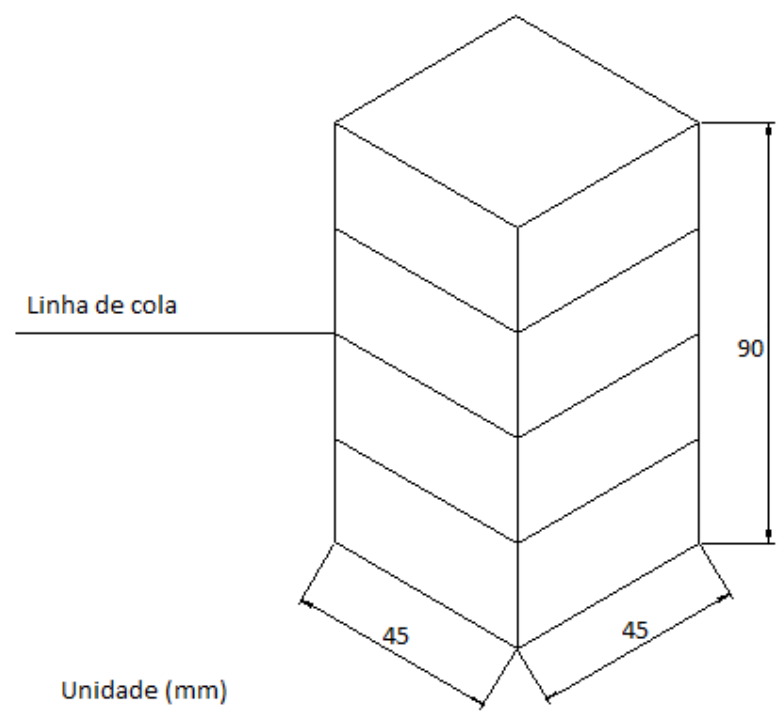

Figura 4. Representação do corpo-de-prova de compressão normal às fibras.

Figure 4. Representation of specimens for normal compression of fibers.

$\mathrm{O}$ ensaio requer seis dias para completar os três ciclos. $\mathrm{O}$ primeiro ciclo totaliza um período de 48 horas (dois dias). No segundo e terceiro ciclo foram repetidas as mesmas etapas do primeiro ciclo, e cada ciclo é composto de duas etapas. Inicialmente, na etapa um do primeiro ciclo, aplicou-se um vácuo de $75 \pm 10 \mathrm{kPa}$ por um período não inferior a $2 \mathrm{~h}$; depois o vácuo foi liberado e aplicou-se água com pressão de $540 \pm 20 \mathrm{kPa}$, também por um período não inferior a $2 \mathrm{~h}$, certificando-se de que os corpos de prova estavam submersos. $\mathrm{Na}$ etapa dois do primeiro ciclo repetiram-se os mesmos procedimentos da etapa um, totalizando $8 \mathrm{~h}$ de permanência na autoclave.

Após o período de ensaios na autoclave, as amostras de aproximadamente $75 \mathrm{~mm}$ de comprimento são colocadas para secar ao ar livre durante $36 \mathrm{~h}$ à temperatura de $28 \pm 2{ }^{\circ} \mathrm{C}$, visando a redução de seu peso, na faixa não superior a 5 a $6 \%$ do peso inicial da amostra. Recomenda-se que a delaminação 
total das linhas de cola, de cada corpo-de-prova, não deva exceder $10 \%$ do comprimento total no topo dos corpos de prova, para que a MLC seja aprovada para o uso exterior, conforme AITC T110 (2007).

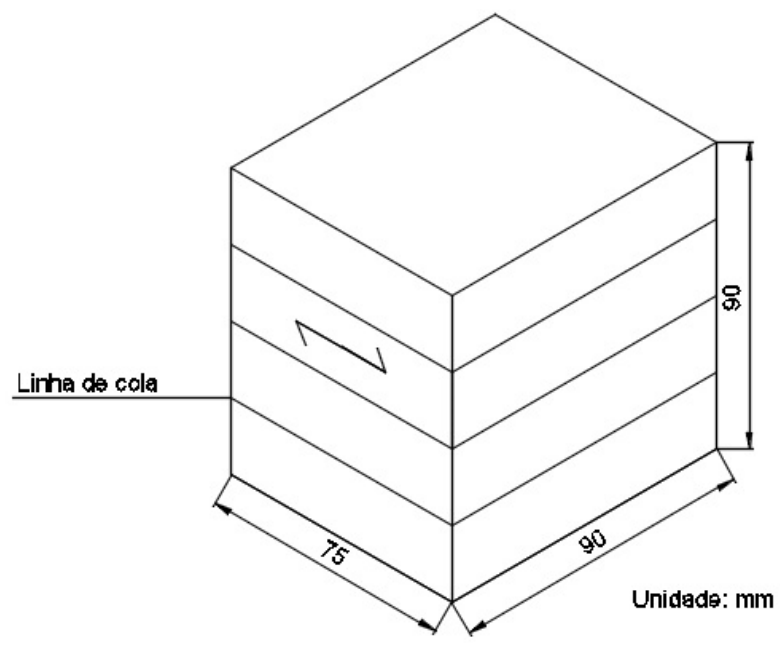

Figura 5. Corpo-de-prova de delaminação.

Figure 5. Specimens for delamination tests.

\section{Visualização da interface madeira-adesivo}

Para a visualização da interface madeira-adesivo, foram retiradas 3 amostras de cada tratamento, da posição transversal à linha de cola. As amostras (cubos) foram fervidas em um béquer durante $8 \mathrm{~h}$ e posteriormente seccionados no micrótomo de deslize, modelo Jung SM 2000, com espessura de 18 micrômetros.

Foi realizado um corte de cada amostra, e cada um deles foi fixado em lâminas provisórias para análise imediata no microscópio óptico, com fotomicrografias, usando o software Axio Vision LE. Foram realizadas 50 medições ao longo da linha de cola para obtenção da espessura média em cada corte.

\section{Corpo de prova estrutural da MLC}

As dimensões do corpo-de-prova estrutural de compressão normal às fibras, obtidas a partir dos elementos de MLC para realização dos ensaios saturados, podem ser verificadas na Figura 6. O procedimento de ensaio na máquina universal de ensaios foi realizado conforme a norma
ASTM D143 - Standard Test Methods for Small Clear Specimens of Timber (ASTM, 2000), pois não há norma que comtemple esse tipo de corpo-de-prova em MLC e porque os resultados serão comparados com os valores obtidos em corpos de prova padronizados nessas normas. A resistência $\left(\mathrm{f}_{\mathrm{c} 90}\right)$ e o módulo de elasticidade $\left(\mathrm{E}_{\mathrm{c} 90}\right)$ à compressão normal às fibras foram obtidos de acordo com as normas ISO 13910 (2005) e NBR 7190 (ABNT, 1997), respectivamente.

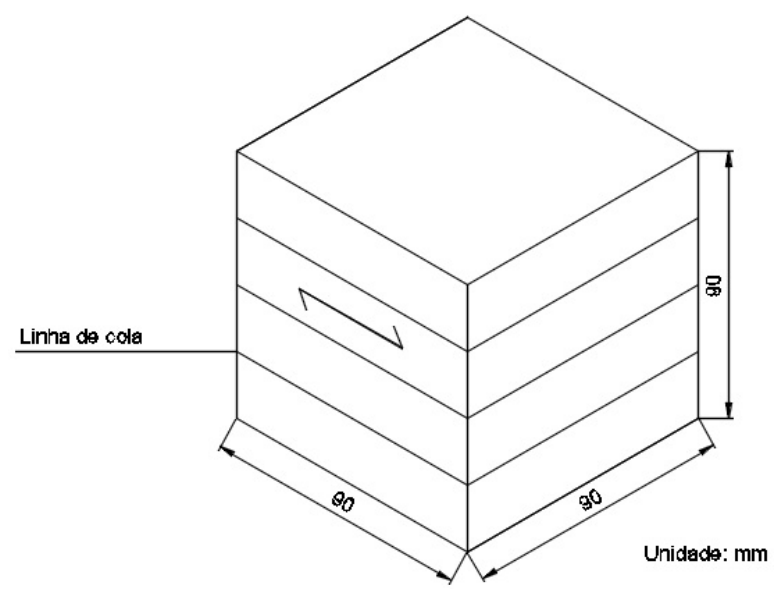

Figura 6. Corpo-de-prova estrutural para ensaio de compressão normal às fibras.

Figure 6. Structural specimens for testing of normal compression of fibers.

\section{Análise Estatística}

O trabalho apresenta os tratamentos L1, L2, MLC-PURL1 e MLC-RFF-L2 sendo o número de repetições igual a dez (10) para cada tratamento nos ensaios, exceto para a tomada de espessura de linha de cola que variou entre 42 e 60 medições (repetições) e no ensaio de compressão normal às fibras dos corpos de prova estruturais que foram 6 (seis) repetições. Para a realização da análise de variância (ANOVA) considerando o delineamento inteiramente casualizado, os dados das variáveis descritas em material e métodos foram submetidos ao teste de normalidade (SHAPIRO; WILK, 1965) e de homogeneidade de variâncias F máximo (HARTLEY, 1950). 
Para comparação das médias foram utilizados o teste de Tukey (mais de duas médias), e o teste F, nos casos em que havia somente dois tratamentos a serem comparados. O nível de significância adotado foi de 5\% de probabilidade de errp. Os dados referentes à falha na madeira e espessura de linha de cola, foram avaliados somente por meio de estatística descritiva, uma vez que as pressuposições para ANOVA não foram atendidas.

\section{Resultados e Discussão}

\section{Propriedades físicas da madeira}

Os valores médios das propriedades físicas, densidade aparente $\left(\rho_{\mathrm{ap}}\right)$, teor de umidade $(\mathrm{U})$, densidade básica $\left(\rho_{\text {bas }}\right)$, retração volumétrica $(\mathrm{RV})$ e inchamento volumétrico (IV) dos dois lotes da madeira de Eucalyptus sp. estão apresentados na Tabela 1 .

Os lotes de madeira de Eucalyptus sp. apresentaram densidades cujas médias não diferiram significativamente ( $p>0,05)$, conforme Tabela 1. O conhecimento das propriedades físicas dos lotes de madeira é fator fundamental para a fabricação de MLC e assim garantir a homogeneidade do material. A umidade pode interferir na colagem visto que tal variação relacionada a retração volumétrica e o inchamento volumétrico provoca deformações excessivas e consequentemente a perda de eficiência da linha de cola. A densidade interfere na pressão e tempo de prensagem dos elementos de MLC porque madeiras com densidades próximas a $1,0 \mathrm{~g} / \mathrm{cm}^{3}$ ou acima desse valor tendem a ter maior dificuldade de colagem.

\section{Propriedades mecânicas da madeira maciça e da}

$M L C$

Os valores de resistência e módulo de elasticidade, propriedades mecânicas, foram encontrados a partir dos resultados obtidos pelos testes de compressão paralela às fibras, cisalhamento na madeira maciça e na linha de cola, e compressão normal às fibras. Foi realizada uma comparação dos valores de compressão paralela às fibras dos corpos de prova de madeira maciça com os corpos de prova feitos a partir dos elementos de MLC secos e saturados, conforme apresentados na Tabela 2.

$\mathrm{Na}$ Tabela 2, as amostras de madeira seca do tratamento L1 apresentaram maior valor de resistência à compressão paralela às fibras do que L2. Os valores das resistências à compressão paralela às fibras para as amostras coladas com os adesivos PUR e RFF não diferiram significativamente ( $p$ > $0,05)$.

Na Tabela 2 para a madeira saturada, os tratamentos não diferiram significativamente $(\mathrm{p}>0,05)$ entre si, apesar de o tratamento colado com RFF ter apresentado valor médio numericamente inferior aos demais tratamentos.

Os valores médios obtidos no ensaio de cisalhamento na madeira maciça (L1 e L2), na linha de cola (PUR e RFF) e porcentagem de falha na madeira seca e saturada estão apresentados na Tabela 3.

Tabela 1. Valores médios das propriedades físicas da madeira de Eucalyptus sp..

Table 1. Average values of physical properties of Eucalyptus sp. wood.

\begin{tabular}{cccccc}
\hline Tratamentos & $\rho_{\text {ap }}\left(\mathbf{g} / \mathbf{c m}^{\mathbf{3}}\right)$ & $\mathbf{U}(\%)$ & $\rho_{\text {bas }}\left(\mathbf{g} / \mathbf{c m}^{\mathbf{3}}\right)$ & $\mathbf{R V}(\%)$ & IV (\%) \\
\hline L1 & $0,635 \mathrm{a}$ & 11,80 & $0,509 \mathrm{a}$ & $13,16 \mathrm{~b}$ & $15,19 \mathrm{~b}$ \\
$\mathrm{~L} 2$ & $0,682 \mathrm{a}$ & 14,33 & $0,539 \mathrm{a}$ & $15,80 \mathrm{a}$ & $18,80 \mathrm{a}$ \\
\hline $\mathrm{CV}(\%)$ & 8,20 & - & 8,15 & 10,80 & 12,60 \\
\hline
\end{tabular}

\footnotetext{
${ }^{*}$ Médias seguidas por mesma letra, na coluna, não diferem estatisticamente pelo teste $\mathrm{F}$ ( $\left.\mathrm{p}>0,05\right)$; $\rho_{\text {ap }}$ - densidade aparente; $\rho_{\text {bas }}-$ densidade básica; L1 - Lote de madeira utilizada para colagem com poliuretano; L2 - Lote de madeira utilizada para colagem com resorcinol-fenolformaldeído; RV - Retração volumétrica e IV - Inchamento volumétrico; CV (\%) - Coeficiente de variação experimental.
} 
Tabela 2. Valores médios de resistência obtidos no ensaio de compressão paralela às fibras.

Table 2. Average values of strength obtained in the compression test parallel to the fibers.

\begin{tabular}{ccccc}
\hline \multirow{2}{*}{ Tratamentos } & \multicolumn{2}{c}{ Seca } & \multicolumn{2}{c}{ Saturada } \\
\cline { 2 - 5 } & $\mathbf{f}_{\mathrm{c} 0, \mathbf{m}}(\mathbf{M P a})$ & $\mathbf{U}(\%)$ & $\mathbf{f}_{\mathrm{c} 0, \mathrm{~m}}(\mathbf{M P a})$ & $\mathbf{U}(\%)$ \\
\hline L1 & $54,49 \mathrm{a}$ & 12,34 & $30,32 \mathrm{a}$ & 68,37 \\
L2 & $47,77 \mathrm{~b}$ & 13,46 & $28,52 \mathrm{a}$ & 60,26 \\
MLC-PUR-L1 & $50,90 \mathrm{~b}$ & 12,43 & $30,98 \mathrm{a}$ & 57,77 \\
MLC-RFF-L2 & $48,15 \mathrm{~b}$ & 14,70 & $27,98 \mathrm{a}$ & 66,55 \\
CV (\%) & 8,32 & - & 10,78 & - \\
\hline
\end{tabular}

${ }^{*}$ Médias seguidas por mesma letra, na coluna, não diferem entre si pelo teste de Tukey ( $\mathrm{p}>0,05$ ); PUR - Poliuretano; RFF - Resorcinol-fenolformaldeído; $\mathrm{f}_{\mathrm{c} 0, \mathrm{~m}}$ - Compressão paralela às fibras; $\mathrm{CV}(\%)$ - Coeficiente de variação experimental.

$\mathrm{Na}$ Tabela 3, a resistência ao cisalhamento nas madeiras maciças e na linha de cola (PUR) não diferiram significativamente $(p>0,05)$ para a condição seca. Tais resultados indicam que os tratamentos foram em sua maioria homogêneos e que o adesivo RFF teve o melhor na condição saturada e o adesivo PUR teve o melhor desempenho na condição seca.

Para o ensaio de cisalhamento na linha de cola, o tratamento com RFF apresentou maior eficiência devido à grande porcentagem de cisalhamento na madeira, tanto na MLC seca, quanto na MLC saturada, sendo o contrário do que ocorreu na linha de cola no tratamento com PUR, conforme a Tabela 3. Logo, o tratamento com RFF seria o mais indicado para ser utilizado em MLC com madeira de Eucalyptus sp. de acordo com os resultados apresentados na Tabela 3.

Na Tabela 4 têm-se os valores médios de resistência e de módulo de elasticidade obtidos por meio do ensaio de compressão normal às fibras.

Tabela 3. Valores médios de resistência obtidos no ensaio de cisalhamento na madeira de Eucalyptus sp. maciça, na linha de cola e porcentagem de falha na madeira de Eucalyptus sp..

Table 3. Average values of strength obtained in tests of shear of solid Eucalyptus sp. wood, glue line and percentage of failure of Eucalyptus sp. wood.

\begin{tabular}{|c|c|c|c|c|c|c|}
\hline \multirow{2}{*}{ Tratamentos } & \multicolumn{3}{|c|}{ Seca } & \multicolumn{3}{|c|}{ Saturada } \\
\hline & $\mathrm{f}_{\mathrm{v} 0, \mathrm{~m}}(\mathrm{MPa})$ & FM (\%) & $\mathrm{U}(\%)$ & $\mathrm{f}_{\mathrm{v} 0, \mathrm{~m}}(\mathrm{MPa})$ & FM (\%) & U (\%) \\
\hline L1 & $11,91 \mathrm{ab}$ & - & 11,41 & $8,88 \mathrm{ab}$ & - & 72,02 \\
\hline $\mathrm{L} 2$ & $11,07 \mathrm{ab}$ & - & 15,38 & $7,37 \mathrm{bc}$ & - & 75,03 \\
\hline MLC-PUR-L1 & $12,68 \mathrm{a}$ & 79,58 & 11,08 & $5,99 \mathrm{c}$ & 13,90 & 88,42 \\
\hline MLC-RFF-L2 & $10,40 \mathrm{~b}$ & 93,50 & 15,23 & $8,96 \mathrm{a}$ & 64,30 & 77,97 \\
\hline CV (\%) & 13,11 & - & - & 16,60 & - & - \\
\hline
\end{tabular}

${ }^{*}$ Médias seguidas por mesma letra, na coluna, não diferem entre si pelo teste de Tukey ( $\left.p>0,05\right)$; fvo,m - Resistência ao cisalhamento; FM (\%) - Porcentagem de falha na madeira; CV (\%) - Coeficiente de variação experimental.

Tabela 4. Valores médios de resistência e de módulo de elasticidade obtidos no ensaio de compressão normal às fibras para os dois tratamentos da MLC de Eucalyptus sp..

Table 4. Average values of strength and of modulus of elasticity obtained in the test of normal compression to the fibers for both treatments of Eucalyptus sp. Glulam.

\begin{tabular}{c|ccc|ccc}
\hline \multirow{2}{*}{ Tratamentos } & \multicolumn{3}{|c|}{ Seca } & \multicolumn{3}{c}{ Saturada } \\
\cline { 2 - 8 } & $\mathbf{f}_{\mathbf{c 9 0 , m}}(\mathbf{M P a})$ & $\mathbf{E}_{\mathbf{c} 90, \mathbf{m}}(\mathbf{M P a})$ & $\mathbf{U}(\%)$ & $\mathbf{f}_{\mathbf{c 9 0 , m}}(\mathbf{M P a})$ & $\mathbf{E}_{\mathbf{c 9 0 , m}}(\mathbf{M P a})$ & $\mathbf{U}(\mathbf{\%})$ \\
\hline MLC-PUR-L1 & $10,61 \mathrm{a}$ & $493,00 \mathrm{a}$ & 11,27 & $6,71 \mathrm{a}$ & $179,65 \mathrm{a}$ & 85,28 \\
MLC-RFF-L2 & $8,68 \mathrm{~b}$ & $476,75 \mathrm{a}$ & 14,80 & $6,28 \mathrm{a}$ & $178,89 \mathrm{a}$ & 81,02 \\
\hline CV (\%) & 8,69 & 15,33 & - & 7,22 & 24,18 & - \\
\hline
\end{tabular}

${ }^{*}$ Médias seguidas por mesma letra, na coluna, não diferem estatisticamente pelo teste $\mathrm{F}(\mathrm{p}>0,05) ; \mathrm{f}_{\mathrm{c} 90, \mathrm{~m}}$ - Resistência média à compressão normal às fibras; $\mathrm{E}_{\mathrm{c} 90, \mathrm{~m}}$ - Módulo de elasticidade médio à compressão normal às fibras; CV (\%) - Coeficiente de variação experimental. 
As madeiras coladas com RFF apresentaram resistência à compressão normal às fibras significativamente $(\mathrm{p}<0,05)$ inferior às coladas com PUR na condição seca; mas não houve diferença significativa $(p>0,05)$ na condição saturada, conforme a Tabela 4. Os valores de resistência a compressão normal às fibras não deveriam a apresentar diferença significativa tanto na condição seca, quanto saturado. Porém, na condição seca houve diferença significativa a qual não foi explicar se ocorreu devido à falta de homogeneidade ou diferença entre umidades das madeiras nos lotes L1 e L2.

As madeiras coladas com RFF apresentaram módulo de elasticidade à compressão normal às fibras que não diferiram significativamente $(p>0,05)$ das coladas com PUR, tanto na condição seca, quanto na condição saturada, conforme a Tabela 4. Tais resultados comprovam a homogeneidade entre as madeiras L1 e L2 e que a madeira saturada perde mais da metade da sua rigidez quando comparada com a madeira seca. $\mathrm{O}$ adesivo e consequentemente a colagem tem influência mínima nesse tipo de ensaio.

O valor médio da delaminação nos corpos de prova colados com PUR foi igual a 34,40\%, enquanto para os colados com RFF foi igual a 5,02\%. Na Figura 7, é possível visualizar o estado final dos corpos de prova após o ensaio de delaminação, apresentando aberturas na linha de cola. Diante de tais resultados é possível vincular o valor maior de delaminação com a espessura menor da linha de cola do PUR, sendo que os resultados de menor delaminação podem ser associados a maior espessura de linha de cola do RFF, conforme apresentados na Figura 8 e na Tabela 5.

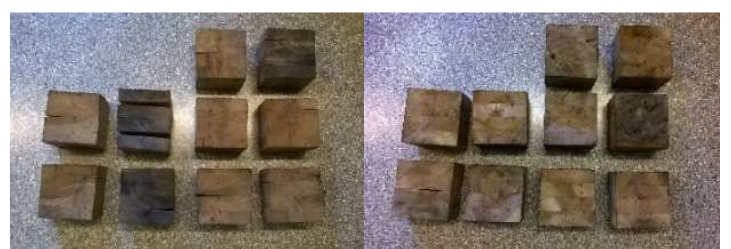

Figura 7. Corpos de prova colados com PUR e RFF, respectivamente.

Figure 7. Specimens glued with PUR and RPF, respectively.
Na Figura 8 vê-se a interface madeira-adesivo das juntas coladas com o adesivo poliuretano (PUR) e com resorcinolfenol-formaldeído (RFF). Observa-se que a linha de cola do tratamento PUR apresentou menor espessura que a do RFF; os valores médios de espessura estão indicados na Tabela 7 .

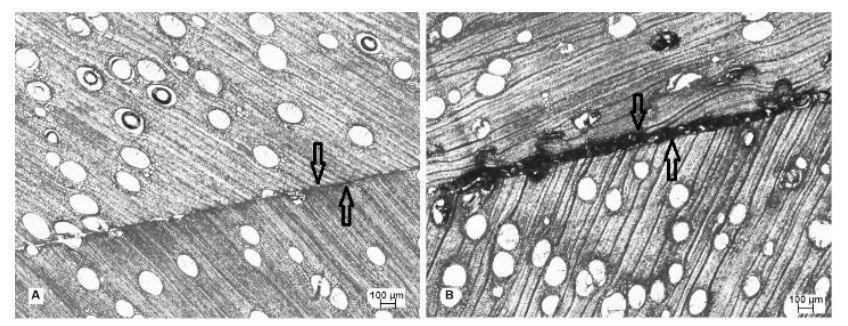

Figura 8. Corte transversal em relação às linhas de cola: (A) - Linha de cola tratamento PUR e (B) - Linha de cola tratamento RFF.

Figure 8. Cross section in relation to the glue lines: (A) - Glue line PUR treatment, and (B) - Glue line RPF treatment.

$\mathrm{Na}$ Tabela 5 têm-se os valores das espessuras médias das linhas de cola dos tratamentos da MLC.

As superfícies coladas com RFF apresentaram uma linha de cola mais eficiente e espessa do que aquelas coladas com PUR; certamente a maior penetração de RFF na madeira foi um dos fatores que proporcionou maior eficiência, conforme mostrado na Tabela 5.

Tabela 5. Valores médios de espessuras das linhas de cola dos tratamentos da MLC.

Table 5. Average values thickness of glue lines of treatments of Glulam.

\begin{tabular}{ccc}
\hline Tratamento & Espessura $(\boldsymbol{\mu m})$ & CV $(\%)$ \\
\hline MLC-PUR-L1 & 24,25 & 41,09 \\
MLC-RFF-L2 & 80,31 & 32,78 \\
\hline
\end{tabular}

Na Tabela 6 têm-se os valores médios para a resistência e módulo de elasticidade obtidos no ensaio de compressão normal às fibras a partir dos corpos de prova estruturais colados com PUR e RFF, ambos na condição saturada. 
Tabela 6. Valores médios de resistência e módulo de elasticidade obtida no ensaio de compressão normal às fibras em corpos de prova estruturais na condição saturada da MLC. Table 6. Average values of strength and modulus of elasticity obtained in the normal compression test to the fibers in structural test specimens in the saturated condition of the Glulam.

\begin{tabular}{cccc}
\hline Tratamentos & $\mathbf{f}_{\mathbf{c 9 0 , m}}(\mathbf{M P a})$ & $\mathbf{E}_{\mathbf{c} \mathbf{9}, \mathbf{m}}(\mathbf{M P a})$ & $\mathbf{U}(\%)$ \\
\hline MLC-PUR-L1 & $5,93 \mathrm{a}$ & $185,22 \mathrm{a}$ & 71,73 \\
MLC-RFF-L2 & $5,66 \mathrm{a}$ & $166,03 \mathrm{a}$ & 61,22 \\
\hline CV $(\%)$ & 5,09 & 12,49 & -
\end{tabular}

* Médias seguidas por mesma letra, na coluna, não diferem estatisticamente pelo teste $\mathrm{F}(\mathrm{p}>0,05) ; \mathrm{f}_{\mathrm{c} 90, \mathrm{~m}}$ - Resistência média à compressão normal às fibras; $\mathrm{E}_{\mathrm{c} 90, \mathrm{~m}}$ - Módulo de elasticidade médio à compressão normal às fibras; CV (\%) - Coeficiente de variação experimental.

As madeiras coladas com RFF apresentaram resistência e módulo de elasticidade à compressão normal às fibras 4,55\% e 10,36\%, respectivamente, inferior àquelas coladas com PUR, mas não houve diferença significativa $(\mathrm{p}>0,05)$ entre os dois tratamentos, conforme a Tabela 6. Os resultados obtidos no ensaio de compressão normal às fibras sofrem pouca influência do adesivo porque a integridade da linha de cola dos corpos de prova foi mantida durante o experimento, devido à não exigência efetiva do adesivo nesse ensaio. Os valores apesentados na Tabela 6 são próximos daqueles apresentados na Tabela 4 quando tomado na mesma condição de umidade, saturação.

\section{Conclusões}

A partir dos resultados apresentados nesse trabalho, pode-se concluir que:

Os resultados obtidos nos ensaios mecânicos para a madeira de Eucalyptus sp. indicam a possibilidade de utilização na fabricação de elementos estruturais de MLC, principalmente na condição seca com PUR e RFF.

A madeira de Eucalyptus sp. associada ao adesivo RFF possui grande potencial no para a fabricação de MLC, inclusive para aplicação na condição saturada, pois os resultados obtidos nos ensaios de delaminação respaldam que é possível a utilização em ambiente externo sujeito a intempéries.

A madeira de Eucalyptus sp. mesmo sendo de ciclo curto, na faixa de 7 a 10 anos de idade, apresentou propriedades mecânicas de resistência que permitem ser utilizadas na fabricação de elemento de MLC para diversos setores, como construção civil e movelaria.

Constatou-se que a utilização de madeiras de reflorestamento de Eucalyptus sp. não só é possível, como necessária para impulsionar o crescimento das estruturas de madeira em MLC no Brasil. No entanto, as pesquisas a respeito de MLC de Eucalyptus sp., especialmente em ambiente saturado, ainda estão em fase inicial. Constata-se que tal desenvolvimento na MLC para essas condições de uso precisará de investimentos em pesquisa.

\section{Agradecimentos}

Os autores agradecem pelo apoio do Programa Institucional de Iniciação Cientifica (PIIC) da Universidade Federal do Espírito Santo (UFES), ao Conselho Nacional de Desenvolvimento Científico e Tecnológico (CNPq) pela concessão da bolsa de iniciação científica, e à Fundação de Amparo à Pesquisa do Espírito Santo (FAPES) pelo financiamento da pesquisa.

\section{Referências}

AITC - AMERICAN INSTITUTE OF TIMBER CONSTRUCTION. AITC T110 - Test methods for structural glued laminated timber - Cyclic delamination test. Centennial, CO, 2007.

APA - AMERICAN PLYWOOD ASSOCIATION. The Engineered Wood Association. Glulam product guide. Tacoma, Washington, EUA, 2008. 32p.

ASTM - AMERICAN SOCIETY FOR TESTING AND MATERIALS. ASTM D 143 - Standard test methods for small clear specimens of timber. Philadelphia, PA, 2000. 
ASTM - AMERICAN SOCIETY FOR TESTING AND MATERIALS. ASTM D 905 - Standard test method for strength properties of adhesive bonds in shear by compression loading. Philadelphia, PA, 2009.

ABNT - ASSOCIAÇÃO BRASILEIRA DE NORMAS TÉCNICAS. Ensaios físicos e mecânicos da madeira - MB26/40. Rio de Janeiro, 1940. 16p.

ABNT - ASSOCIAÇÃO BRASILEIRA DE NORMAS TÉCNICAS. NBR 7190. Projeto de Estruturas de Madeira: 1997. Rio de Janeiro, 1997.

ICIMOTO, F. H.; FERRO, F. S.; CALIL JUNIOR, C. Dormentes de Madeira Laminada Colada de Pinus oocarpa. In: XV Encontro Brasileiro em Madeiras e Estruturas de Madeira, 15., 2016, Curitiba. Anais... Curitiba: IBRAMEM, 2016.

HARTLEY, H. O. The use of range in analysis of variance. Biometrika. London, v. 37, p. 271-280, 1950.

ISO - INTERNATIONAL STANDARD ORGANIZATION. ISO 13910. Structural timber - Characteristic values of strength-graded timber - Sampling, full-size testing and evaluation. Switzerland, 2005. 30p

SHAPIRO, S. S.; WILK, M. B. An analysis of variance test for normality (Complete sample). Biometrika, London, v. 52, p. 591-611, 1965.

TEREZO, R. F.; SZÜCS, C. A. Análise de desempenho de vigas em madeira laminada colada de parica (Schizolobium Amazonicum Huber ex. Ducke). Scientia Forestalis, Piracicaba, v. 38, n. 87, p. 471-480, set. 2010. 